\title{
A Novel Combined Modelling and Optimization Technique for Microwave Components
}

\author{
Yanghua GAO, Zhihua ZHANG, Hailiang LU
}

\begin{abstract}
This paper presents a novel combined parametric modelling and design optimization technique for microwave components utilizing the neural networks. The proposed technique provides an iterative mechanism between ANN model training and design optimization update. This iterative mechanism is fully automated and requires no manual intervention. Furthermore, the proposed technique overcomes the limitations of the common ANN optimization strategy where the fixed training region of the ANN model limits the freedom of design optimization. The proposed technique automatically enlarges the ANN training region until an optimization solution satisfying the user's design specification is met. Once the whole iterative process is finished, an accurate parametric model and an optimal solution satisfying the design specification are simultaneously generated. A parametric modelling and design optimization example of a wideband QuasiElliptic filter design is presented to demonstrate the validity of this technique.
\end{abstract}

Keywords: Automatic Neural Networks; design optimization; microwave components; parametric modelling

\section{INTRODUCTION}

Artificial neural network (ANN) techniques are an important development in electromagnetic (EM)-based modelling and optimization of microwave components [1]-[4]. The most common strategy for ANN based design optimization consists of generating an ANN model of the microwave problem with a certain training region of the design parameters, and then applying gradient-based optimization to the trained ANN model to find the optimal solution that yields the desired responses [5]. This ANN based microwave design is more powerful and faster than direct EM optimization. However, the fixed and pre-set training region of the ANN model may limit the optimization search and prevent the optimization from reaching the user's design specification. It is also cumbersome to manually re-define the training data range and re-perform ANN training for different design specifications.

This paper presents a combined parametric modelling and design optimization technique for microwave components. This technique provides an iterative mechanism to refine the ANN model and update the design optimization. The iterative mechanism requires no manual intervention and is fully automated: parallel and automatic data generation, automatic training and optimization, and automatic generation of an acceptable solution satisfying the user's design specification. Furthermore, the training region of the ANN model is adaptive and changes according to the needs of design optimizations. If it is impossible to find an acceptable solution for the user's design specification within the current training region, the proposed optimization technique will automatically enlarge the ANN training range. Once the whole iterative process is finished, the proposed technique can produce an accurate parametric ANN model and simultaneously generates an acceptable optimization solution satisfying user's design specification. The validity of the proposed approach is confirmed by an example of a wideband QuasiElliptic filter modelling and design.

\section{FORMULATION OF THE COMBINED PARAMETRIC MODELLING AND DESIGN OPTIMIZATION TECHNIQUE}

Contrary to the step-by-step approach, the proposed combined parametric modeling and design optimization technique focuses on the mutual dependence of various subtasks like data generation, neural network training, model optimization update and verification. Our algorithm links these subtasks through neural-network learning phenomena and integrates them into one unified process. The integrated process is computerized and is carried out automatically in a stage-wise fashion. Here one cycle of ANN training and subsequent design optimization using the trained ANN model is defined as a stage. The overall iterative process runs stage by stage with ANN training and optimization update in each stage. Within a stage, the algorithm facilitates periodic communication between various subtasks, thus enabling adjustment or enhancement in the execution of a subtask based on the feedback from other subtasks. The algorithm has built-in simulation drivers (e.g., CST driver, HFSS driver and ADS driver) for facilitation of automatic data generation during ANN modeling and optimization process. Automation of the combing model development and design optimization process shifts the workload from human to computer thus making model development more efficient and speeding up the design optimization.

We define $\boldsymbol{L}^{k}$ as training data set during the $k^{\text {th }}$ stage, i.e., $\boldsymbol{L}^{\boldsymbol{k}}=\left\{\boldsymbol{x}_{\mathrm{i}} \mid\left(\boldsymbol{x}_{\mathrm{i}}, \boldsymbol{y}_{\mathrm{i}}\right)\right.$ is a training sample $\}$. Training data sets $\boldsymbol{L}^{k}$ are updated in every stage. Let $S^{k}$ represent neuralnetwork structure in the $k^{\text {th }}$ stage with outputs $\boldsymbol{y}^{\boldsymbol{k}}=f_{\mathrm{ANN}}(\boldsymbol{x}$, $\left.w^{k}\right)$, where $\boldsymbol{w}^{k}$ is the corresponding ANN internal weights. Let $\boldsymbol{R}^{k}$ represent the training and optimization range during the $k^{\text {th }}$ stage. For a given microwave design problem, firstly check if there is an existing ANN model for this design. In the initial stage, the ANN model may not be accurate enough due to the limited training data. However as the number of stages increases, the ANN model becomes more accurate as more training data are generated and used to train the ANN model. Thus the design optimization becomes faster. The computational process is described by the flowchart in Fig. 1. Various steps involved in the proposed technique are summarized into a computational algorithm below. 
Case1: If an ANN model does not exist.

Step 1) At the initial stage, .i.e, $k=0$, generate the training data $\boldsymbol{L}^{\mathbf{0}}$ within user's specified range $\left[\boldsymbol{x}^{\mathbf{0}}{ }_{\text {min }} \boldsymbol{x}^{\mathbf{0}}{ }_{\text {max }}\right]$ of design variables by driving multiple EM simulations in parallel. Partial composite design of experiments method is used to determine the size and distribution of the training data. The training and optimization region is as

$$
R^{0}=\left\{\boldsymbol{x} \mid \boldsymbol{x}_{\min }^{0} \leq \boldsymbol{x} \leq \boldsymbol{x}_{\max }^{0}\right\}
$$

where $\boldsymbol{x}_{\text {min }}^{\mathbf{0}}$ and $\boldsymbol{x}_{\text {max }}^{\mathbf{0}}$ are the extreme boundaries of $\boldsymbol{R}^{\mathbf{0}}$. Initialize an MLP neural network $f_{\mathrm{ANN}}\left(\boldsymbol{x}, \boldsymbol{w}^{\mathbf{0}}\right)$ [6]. Here $\boldsymbol{x}$ represent the inputs of the ANN model, i.e., design parameters, and $\boldsymbol{w}^{\mathbf{0}}$ represent the ANN internal weights in the initial stage.

Step 2) At the $k^{\text {th }}$ stage, perform an error-based ANN training to learn the training data $\boldsymbol{L}^{\boldsymbol{k}}$ generated. The ANN model is trained through adjusting the ANN internal weights $\boldsymbol{w}^{k}$, such that the difference between the available training data $\boldsymbol{d}$ and the outputs of the ANN model is minimized

$$
\min _{\boldsymbol{w}^{k}} \sum_{i \in L^{k}}\left\|\boldsymbol{f}_{A N N}\left(\boldsymbol{x}_{i}, \boldsymbol{w}^{k}\right)-\boldsymbol{d}\left(\boldsymbol{x}_{i}\right)\right\|,
$$

In this process, ANN weights $\boldsymbol{w}^{\boldsymbol{k}}$ are variables and ANN inputs $\boldsymbol{x}$ are fixed at the geometrical samples.

Step 3) Perform design optimization using the trained ANN model to find the best solution $\boldsymbol{x}_{\boldsymbol{f}}$ within the current training region $\boldsymbol{R}^{\boldsymbol{k}}$, such that

$$
\boldsymbol{x}_{f}=\arg \min _{x_{f} \in R^{k}} U\left(\boldsymbol{y}\left(\boldsymbol{w}, \boldsymbol{x}_{f}\right)\right),
$$

where $U$ is the minimax objective function expressed in terms of the design specifications. In this process, ANN inputs $\boldsymbol{x}$ are optimization variables and ANN weights $\boldsymbol{w}^{k}$ are fixed. Due to the fast computation speed of ANN models, this optimization procedure is inexpensive. Because of the nature of parametric ANN models, the best solution $\boldsymbol{x}_{\boldsymbol{f}}$ of this design optimization is found in this current global training region.

Step 4) Verify the obtained solution $\boldsymbol{x}_{\boldsymbol{f}}$ through driving EM simulation using the geometrical values obtained from Step 3. Without any extra time other than the time for generating the EM data for verification, multiple additional training data in the neighborhood of the obtained solution are generated simultaneously by driving multiple EM simulations in parallel.

Newly generated data $\boldsymbol{L}^{\text {new }}$ including the EM data used for verification in the current stage are added into the existing training data set. The training index sets are augmented with newly generated data as

$$
L^{k+1}=L^{k} \bigcup L^{\text {new }}
$$

where $L^{k+1}$ are used for the subsequent stage of ANN training and subsequent design optimization. If the responses from EM simulation at $\boldsymbol{x}_{\boldsymbol{f}}$ meet the user's design specification, the whole iteration process is finished. We obtain an accurate parametric model and simultaneously an acceptable solution for the user's design specification. Otherwise, use the obtained solution $\boldsymbol{x}_{\boldsymbol{f}}$ from this step as the starting point for the next stage, i.e., $k=k+1$.

In the subsequent stage, neural network $S^{k+1}$ is trained with samples in $\boldsymbol{L}^{\boldsymbol{k}+\boldsymbol{1}}$. Increase the stage counter by 1 and go to the next step.

Step 5) Check if the number of stages $\boldsymbol{N}_{\text {iter }}$ for the current training data range exceeds the limit $\boldsymbol{N}_{\text {lim }}$, i.e., number of stages per training data range specified by the user. If it exceeds, it means that it is impossible to find an optimal solution satisfying the design specification within the current training range $\boldsymbol{R}^{k}$. Therefore, we enlarge the optimization space beyond the current ANN training range and go to Step 3. The new date range is

$$
R^{k+1}=\left\{\boldsymbol{x} \mid \boldsymbol{x}_{\min }^{k}-\Delta \boldsymbol{x} \leq \boldsymbol{x} \leq \boldsymbol{x}_{\max }^{k}+\Delta \boldsymbol{x}\right\}
$$

where $\Delta \boldsymbol{x}$ represent the incremented data range. Note that the results obtained from the design optimization outside the training region of ANN models may be unreliable. However it shows the directions where we should add new training data. If the number of stages does not exceed the limit, then add the newly generated data into our existing training data set, and go to Step 2.

Case2: If there is a trained ANN model, go to Step 3.

\section{ANALYSIS OF SPEEDUP}

We will use speedup $S_{p}$ to measure the performance of the combined parametric modeling and design optimization technique. Let $T_{g}, T_{r}$ and $T_{o}$ be the data generation time for per geometry, ANN model training time and optimization time for per stages, respectively. Let the speedup $S_{p}$ be the ratio between the execution time of EM internal optimization and the combined parametric modeling and design optimization technique on $P$ processors. For Case 1 where the ANN model does not exist, the speedup $\mathrm{S}_{p}$ as

$$
S_{p}=\frac{T_{g} \cdot N}{T_{g} \cdot n / P+\left(T_{g}+T_{r}+T_{o}\right) \cdot m}
$$

where $n$ is the number of training data generated in the initial stage (the typical value, i.e., 25), $m$ is the number of stages (the typical value, i.e., 15), and $N$ is the number of total optimization iterations for EM internal optimization (the typical value, i.e., 125). Since the ANN model training time $T_{r}$ and optimization time $T_{o}$ for per stage are much less than the data generation time $T_{g}$, the value $S_{p}$ will be much larger than 1 . For Case 2 where there is a trained ANN model, the speedup $\mathrm{S}_{p}$ as

$S_{p}=\frac{T_{g} \cdot N}{\left(T_{g}+T_{r}+T_{o}\right) \cdot m}$,

As shown in Eq. (5), without the need of data generation time $T_{g}$ in the initial stage, the speedup $S_{p}$ would be a much larger value. Much time is saved when 
the parametric model is re-used again and again with different optimization goals.

\section{MODELLING AND DESIGN OPTIMIZATION OF THE QuasiElliptic FILTER}

In this example, we illustrate the proposed combined parametric modeling and design optimization technique for the wideband QuasiElliptic filter design. The QuasiElliptic filter is implemented in a Low-Temperature Co-fired Ceramics process (LTCC). The model development and design optimization are carried out using the Matlab program [7], and the EM simulator used for data generation is HFSS [8].

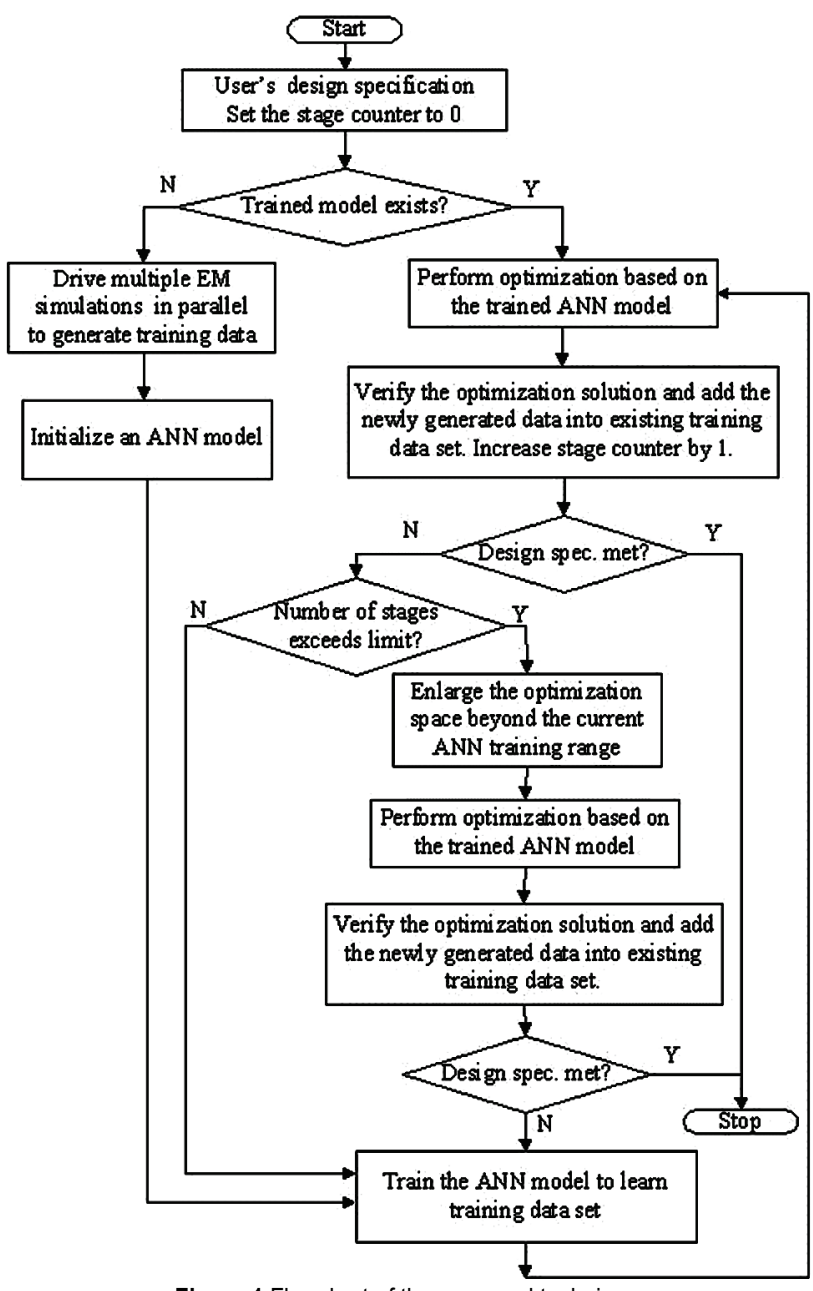

Figure 1 Flowchart of the proposed technique

The QuasiElliptic filter structure (QEBPF) is shown in Fig. 1. This filter having two pairs of transmission zeros gives much improved skirt selectivity and wide bandwidths. The structure of the neural network model for this QEBPF example is shown in Fig. 2. In this example, the neural network model has nine geometrical

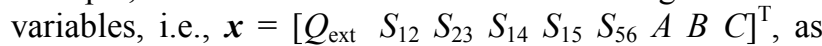
defined in Fig. 1. Frequency is also used as an additional input. The model has four outputs, i.e., $\boldsymbol{y}=\left[R S_{11} I S_{11} R S_{12}\right.$ $\left.I S_{12}\right]^{\mathrm{T}}$, which are composed of the real and imaginary parts of $S_{11}$ and $S_{12}$. Let $\boldsymbol{d}$ be a vector representing the outputs of the HFSS EM simulations. The object of neural network training is to adjust the neural network internal weights such that the error between $y$ and the training data $\boldsymbol{d}$ is minimized.

In order to illustrate the re-use of the parametric model for different design optimizations, we use the proposed technique to design three filters with different frequency bands. The design specifications for these three filters are:

Specification for filter 1: $\left|S_{11}\right| \leq-10 \mathrm{~dB}$ and $\left|S_{21}\right| \geq-3$ $\mathrm{dB}$ at frequency range of $57 \mathrm{GHz}$ to $63 \mathrm{GHz}$.

Specification for filter 2: $\left|S_{11}\right| \leq-10 \mathrm{~dB}$ and $\left|S_{21}\right| \geq-3$ $\mathrm{dB}$ at frequency range of $55 \mathrm{GHz}$ to $61 \mathrm{GHz}$.

Specification for filter 3: $\left|S_{11}\right| \leq-10 \mathrm{~dB}$ and $\left|S_{21}\right| \geq-3$ $\mathrm{dB}$ at frequency range of $62 \mathrm{GHz}$ to $68 \mathrm{GHz}$.

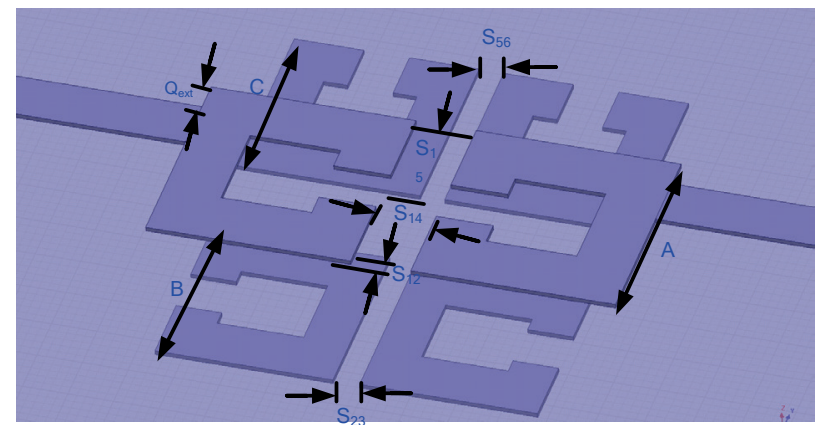

Figure 2 The structure of a QuasiElliptic filter

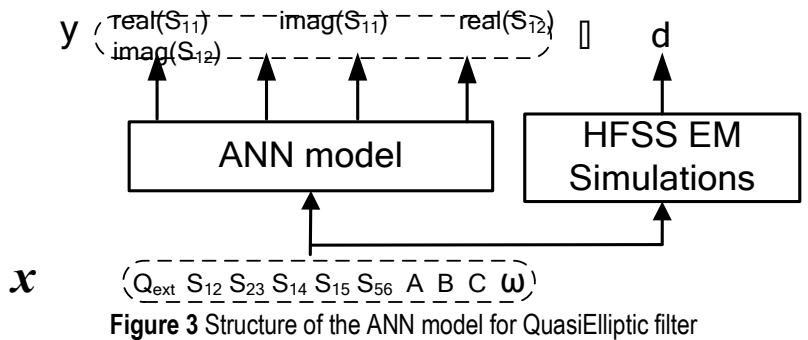

The starting values of five geometrical variables are chosen as $\boldsymbol{x}=\left[\begin{array}{lllllllll}40 & 110 & 60 & 140 & 110 & 60 & 450 & 390 & 410\end{array}\right]^{\mathrm{T}}$ (all values in um). The frequency range used varies from 40 $\mathrm{GHz}$ to $80 \mathrm{GHz}$ with a step size of $0.2 \mathrm{GHz}$. In the initial stage, we generated 32 sets of geometries as the training data, which are within $\pm 10 \%$ of the starting values. The training data are generated by running multiple EM simulations in parallel (with 8 parallel threads). The numbers of stages used for optimization of filter 1, filter 2 and filter 3 are 4, 7 and 8, respectively. In each stage, 5 new sets of training data in the neighborhood of the optimal solution from the previous step are generated simultaneously. For filter 2, even after 5 stages ( 5 is the limit specified by user), we still cannot find an acceptable solution satisfying the specification within the existing training data range. Therefore, the proposed technique expanded the data range to $\pm 15 \%$ of the starting values. For filter 3 , even after 5 stages ( 5 is the limit specified by user), we still cannot find an acceptable solution satisfying the specification within the existing training data range. Therefore, the proposed technique expanded the data range to $\pm 25 \%$ of the starting values.

As shown in Fig. 4, broadband accuracy of the ANN model is confirmed by its good agreement with EM simulation data in terms of magnitude of $S_{11}$ and $S_{21}$. The proposed technique can produce an accurate parametric 
ANN model and simultaneously generates acceptable optimization solutions for the user's two design specifications.

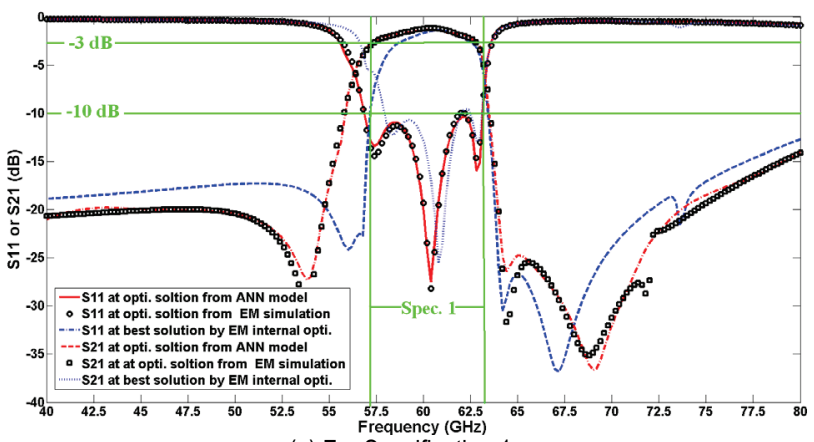

(a) For Specification 1

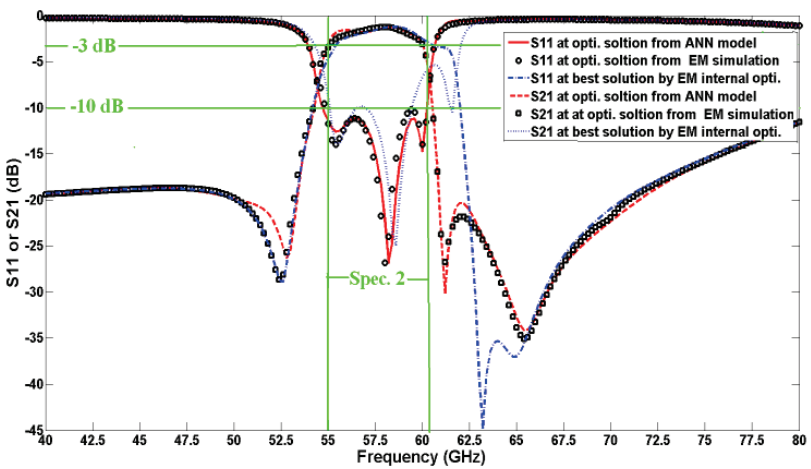

(b) For Specification 2

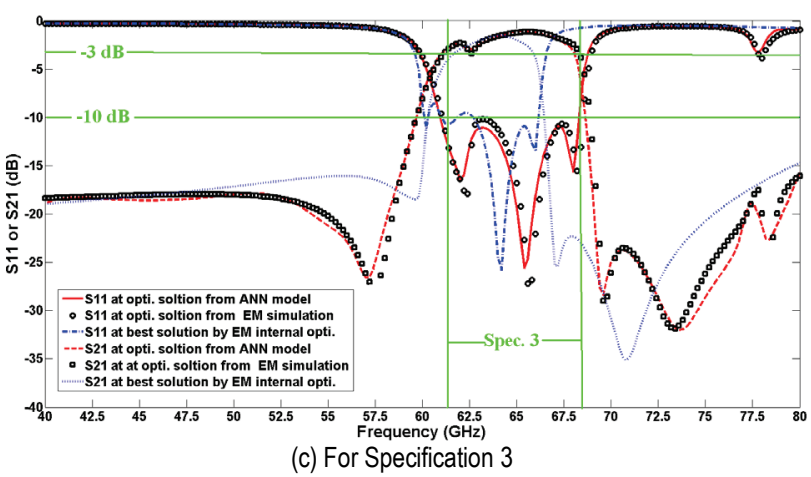

Figure 4 Comparison of the magnitude $\mathrm{S} 11$ and $\mathrm{S} 12$ of the ANN model and HFSS EM data for the QuasiElliptic filter example for different Specifications. (a) For Specification 1; (b) For Specification 2; (c) For Specification 3. As shown in this figure, the proposed technique not only matches very well with HFSS EM solutions but also works substantially faster than HFSS EM optimization

Table 1 CPU time of optimizations for QuasiElliptic filter example

\begin{tabular}{|c|c|c|}
\hline \multirow{2}{*}{$\begin{array}{c}\text { Different } \\
\text { Spec. }\end{array}$} & $\begin{array}{c}\text { HFSS internal } \\
\text { optimizations }\end{array}$ & CPU Time \\
\cline { 2 - 3 } $\begin{array}{c}\text { Spec. for } \\
\text { filter 1 }\end{array}$ & $\begin{array}{c}816 \mathrm{Min} \\
\text { (120 iterations) }\end{array}$ & $\begin{array}{c}\text { O2 Min ( including time for generating } \\
\text { 32 sets of initial training data) } \\
\text { +32 Min (including time for generating } \\
\text { 20 new sets of training data, ANN } \\
\text { training time and optimization time) }\end{array}$ \\
\hline $\begin{array}{c}\text { Spec. for } \\
\text { filter 2 }\end{array}$ & $\begin{array}{c}1002 \text { Min } \\
\text { (168 iterations) }\end{array}$ & $\begin{array}{c}\text { 54.8 Min (including new 35 sets data } \\
\text { generation time, ANN training time and } \\
\text { optimization time) }\end{array}$ \\
\hline $\begin{array}{c}\text { Spec. for } \\
\text { filter 3 }\end{array}$ & $\begin{array}{c}\text { 876 Min } \\
\text { (123 iterations) }\end{array}$ & $\begin{array}{c}\text { 62.6 Min (including time for } \\
\text { generating 40 new sets of training data } \\
\text { ANN training time and optimization } \\
\text { time) }\end{array}$ \\
\hline Total & 2694 Min & 162.4 Min \\
\hline
\end{tabular}

Tab. 1 compares CPU time of our proposed technique and EM internal optimization with the same initial points and three filter design specifications. For this example, we can see that our proposed technique can drastically reduce design cycle and much time is saved when the parametric model is re-used again and again with different filter optimization goals.

\section{CONCLUSION}

A combined parametric modeling and design optimization technique for microwave components based on the neural networks has been presented. The proposed technique automatically expands the ANN training region as demanded by design optimization needs. Once the whole optimization process is finished, an accurate parametric model and an optimization solution satisfying user's design specification are simultaneously generated. As the proposed technique is used for more design specifications, the parametric model becomes more accurate and the design optimization becomes faster. Furthermore, this universal technique is applicable to other microwave component designs, i.e., RFID tag/reader antennas and multiband base station antennas.

\section{Acknowledgements}

This publication was written with support of the Research Program of ZJZY (Grant NO ZJZY2015E006).

\section{REFERENCES}

[1] Cao, Y., Reitzinger, S. \& Zhang, Q. J. (2011). Simple and efficient high dimensional parametric modeling for microwave cavity filters using modular neural network. IEEE Microw. Wireless Compon. Lett., 21(5), 258-260. https://doi.org/10.1109/LMWC.2011.2127465

[2] Cao, Y., Wang, G., Gunupudi, P. \& Zhang, Q. J. (2013). Parametric modeling of microwave passive components using combined neural networks and transfer functions in the time and frequency. Int. J. RF Microw., 23, 20-33. https://doi.org/10.1002/mmce.20630

[3] Abdolrazzaghi, M., Hossein Zarifi, M., Pedrycz, W. \& Daneshmand, M. (2017). Robust Ultra-High Resolution Microwave Planar Sensor Using Fuzzy Neural Network Approach. IEEE Sensors Journal, 17(2), 323-332. https://doi.org/10.1109/JSEN.2016.2631618

[4] Qaddoumi, N. N., El-Hag, A. H. \& Saker, Y. (2014). Outdoor Insulators Testing Using Artificial Neural Network-Based Near-Field Microwave Technique. IEEE Trans. Instrumentation and Measurement, 63(2), 260-266. https://doi.org/10.1109/TIM.2013.2280486

[5] Feng, F., Zhang, C., Ma, J. \& Zhang, Q. J. (2016). Parametric modeling of EM behavior of microwave components using combined neural networks and poleresidue-based transfer functions. IEEE Trans. Microw. Theory Tech., 64(1), 60-77. https://doi.org/10.1109/TMTT.2015.2504099

[6] Gongal-Reddy, V., Zhang, S., Zhang, C. \& Zhang, Q. J. (2016). Parallel Computational Approach to Gradient Based EM Optimization of Passive Microwave Circuits. IEEE Trans. Microwave Theory Tech. , 64(1), 44-59. https://doi.org/10.1109/TMTT.2015.2504096

[7] MATLAB and Statistics Toolbox Release 2014b, The MathWorks, Inc., Natick, Massachusetts, United States.

[8] HFSS Version 17, ANSYS, Canonsburg, PA, 2016. 


\section{Contact information:}

Yanghua GAO, Senior Engineer. Dr

Corresponding author

Information Center of China Tobacco Zhejiang Industrial Co. Ltd.

No. 77, Zhongshan South Road, Hangzhou 310008, China

E-mail: gaoyh@zjtobacco.com

\section{Zhihua ZHANG, Senior Engineer}

Information Center of China Tobacco Zhejiang Industrial Co. Ltd.

No. 77, Zhongshan South Road, Hangzhou 310008, China

E-mail: zzh@zjtobacco.com

Hailiang LU, Engineer

Information Center of China Tobacco Zhejiang Industrial Co.,Ltd

No. 77, Zhongshan South Road, Hangzhou 310008, China

E-mail: Ih!@zitobacco.com 